\title{
Merging in the Dust
}

Gas-rich and intensely luminous, distant submillimeter galaxies (so termed for their emission wavelength, not their spatial extent) form stars at very high rates. It has been suggested that interactions between the galaxies power the emission, but most studies so far have been marred by the presence of dust, which can obscure or produce misleading signs of interaction. To circumvent this problem, Engel et al. analyzed high-resolution observations of $\mathrm{CO}$ emission lines from 12 submillimeter galaxies, 4 of which were newly observed. The $\mathrm{CO}$ lines trace the molecular gas that fuels star formation, and their observation is not affected by the presence of dust. Of the 12 galaxies analyzed, 5 were double systems, consisting of two galaties engaged in the early stages of interaction; the remaining 7 either showed signs of being at an intermediate stage of merging or were compact galaxies, plausibly the end result of two galaxies coalescing into one. These results indicate that most submillimeter galaxies, if not all, are the product of interactions and mergers of galaxies. - MJC

Astrophys. J. 724, 233 (2010).

BIOPHYSICS

\section{Spiral Enhancement}

When light strikes tiny metal particles, it can excite electrons at their surfaces along a pathway termed a plasmon resonance. Recently this phenomenon has been put to use to enhance Raman scattering by molecules in the vicinity of the particles, often by a factor of a billion or more. Hendry et al. explore a distinct application of plasmon excitation, in which they assemble mirror-image arrays of gold particles that possess a spiral sort of two-dimensional chirality, and as a result interact differently with left and right circularly polarized light. They then deposit chiral proteins onto the surfaces and examine the associated perturbations to circularly polarized plasmon excitation of left- versus right-handed particles (validated by control experiments with achiral particles of similar size and shape). They find that proteins with $\beta$-sheet motifs induce significantly greater shifts to one array's resonances over its counterpart's, whereas those with $\alpha$ helices induce little dissymmetry. Moreover, the effective refractive index shifts extracted from the $\beta$-sheet measurements are roughly a millionfold higher than those associated with differential circularly polarized light scattering by the biomolecules in solution. Though the detailed mechanism underlying the effect remains under study, the authors posit that quadrupolar interactions between the proteins and chiral optical fields at the surface play a key role. - JSY

Nat. Nanotechnol. 5, 10.1038/NNAN0.2010.209

(2010).

PHARMACOLOGY

\section{Switching Drugs}

Despite the long list of side effects that accompany many medications, most people still take their medicine. Perhaps this is because we know there is so much variation in drug effectiveness and side effects among individuals that we hope we will experience the benefits of the drug and be spared the side effects. Although such variation is well known, why is it so? Morris et al. sought to answer this question by comparing the spatial expression pattern of genes encoding 49 common drug targets found in the brain, in several inbred strains of mice. Many of the genes examined encode neuropsychiatric drug targets. Over 15,000 brain sections representing 203 regions of the brain were analyzed. Differences between strains occurred largely at the level of specific cell classes; for example, among neuronal subsets. Over half of the targets showed some type of interstrain variation in which brain structures they were expressed. When expression was examined across strains, strains that were more closely related showed more similar expression patterns. Gene expression in areas of the brain involved in autonomic functions, such as the hypothalamus, brainstem, and medulla, was very conserved. In contrast, the forebrain region, cortex, and hippocampus, which are involved in cognition, learning, and memory, showed the greatest interstrain expres sion variation. Whether such variation in gene expression also occurs in humans, and whether it influences the variation seen in the therapeutic effectiveness of drugs, still needs to be examined. - BAP

Proc. Natl. Acad. Sci. U.S.A. 107, 19049 (2010).

EVOLUTION

\section{Who Needs Sex?}

Despite the advantages conferred by mixing one's genes through sexual reproduction, many organisms, including plants, fungi, and animals, reproduce asexually. Asexual species are generally assumed to be evolutionary dead ends, however, because of the lack of genetic recombination and accumu- 
lation of mutations. As such, they are expected to reach extinction rapidly, which may explain their relative rarity. Stöck et al. examined the evolutionary history of the asexual Amazon Molly, a fish thought to have arisen through hybridization of two parental species. Crosses of their putative ancestors failed to recapitulate the asexual phenotype. A survey of nuclear and mitochondrial loci within the asexual lineages showed considerable genetic variation and suggested a monophyletic origin, which indicates that the Amazon Molly originated from a single, or very small number, of founders. These results suggest that asexual fish species may be rare not because of the deleterious effects of asexuality but rather because the genomic conditions under which they can arise are rare. - $\mathrm{LMZ}$

Mol. Ecol. 10.1111/

j.1365-294X.2010.04869.x (2010).

\section{PSYCHOLOGY}

\section{What's Left Undone Lingers On}

Multitasking has a long history in computer operating systems, from the 1960 s when programs were executed in batches on mainframes to refinements implemented on personal computers a quarter century ago. Swapping resources from one task to another is easy if they are independent, but doing so efficiently when resources need to be shared is more challenging. Fortunately, computers don't seem to experience the kind of interference described by Masicampo and Baumeister. They demonstrate that humans suffer from a hangover due to unfulfilled goals: When people were primed to strive for honesty as a goal and then required to write about an episode in which they had acted dishonestly, the induced sense of incompleteness negatively affected their ability to solve anagrams, a task that relies on fluid intelligence. Neither the prime alone nor the recounting of the episode sufficed, and people who had been primed but then wrote about someone else's dishonesty were not similarly afflicted. Furthermore, the unfulfilled goal, though detectable with implicit measures of activation, did not rise to the level of reportable or conscious awareness. - G]C

J. Exp. Soc. Psychol. 10.1016/ j.jesp.2010.10.011 (2010).

MICROBIOLOGY

\section{Aiding the Enemy}

Paradoxically, some microbial infections get a boost from host immune responses. A group of worm parasites called filarial nematodes, which

cause river blindness and elephantiasis, adapt their development to maximize reproductive success in response to specialized host immune cells called polynuclear eosinophils. Babayan et al. have found that even the most vigorous eosinophil response is rarely completely protective, and in fact seems to invigorate the worms to reproduce earlier in their life cycle with larger numbers of offspring. The problem lies in current strategies for experimental vaccine development, which rely on the same eosinophil responses that send the reproductive signals to the parasite. Hence, seeking to develop an antifilarial vaccine that does not provide sterilizing immunity may not result in public health benefit. - CA

PLoS Biol. 8, e1000525 (2010).

APPLIED PHYSICS

\section{Blast Assessments}

Shrapnel is not the only danger posed by bombs. The exposure of soldiers to blast waves from explosive devices may, like the repeated blows to the head inflicted on boxers in the ring, result in traumatic brain injury that is not always immediately perceptible or diagnosed. As in the case of the punch-drunk boxer, continual exposures of this sort can lead to serious damage in the long term. Cullen et al. have devised a simple method based on the blast-induced change in color of a photonic crystal to provide a quantitative measure

Before
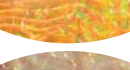

of blast exposure. The

photonic crystal-similar in structure to an opal, with its material disrupted by a periodic array of holes-shimmers with a signature set of colors indicative of its particu-

After lar periodic structure. The authors lithographically

fabricated crystalline strips, which could be attached to helmets and other clothing, from chemically robust resins stable to temperatures as high as $300^{\circ} \mathrm{C}$. Exposure to the shockwaves of an explosion distorts the structure and the color of these photonic crystal sensor strips. Calibration of the color change to blast strength can then provide an instant assessment of blast exposure and the subsequent need for precautions or medical intervention that may otherwise be overlooked. - ISO

Neurolmage 10.1016/ j.neuroimage.2010.10.076 (2010).

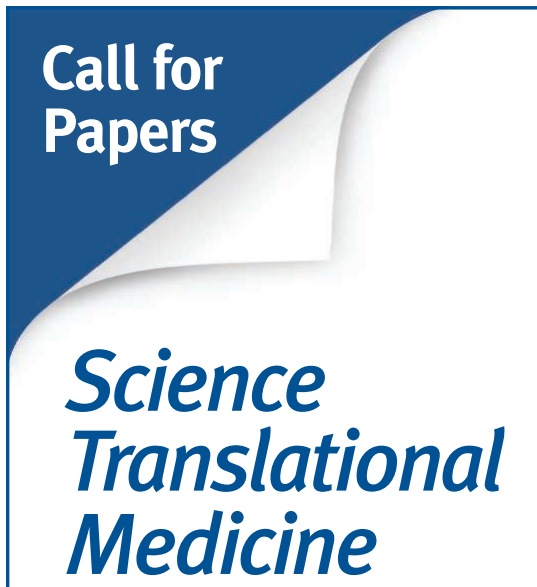

Integrating Medicine and Science

The new journal from the publisher of Science stands at the forefront of the unprecedented and vital collaboration between basic scientists and clinical researchers.

- Cardiovascular Disease

- Neuroscience/Neurology/ Psychiatry

- Infectious Diseases

- Cancer

- Health Policy

- Bioengineering

- Chemical Genomics/ Drug Discovery

- Other Interdisciplinary Approaches to Medicine

\section{Submit your research at www.submit2scitranslmed.org}

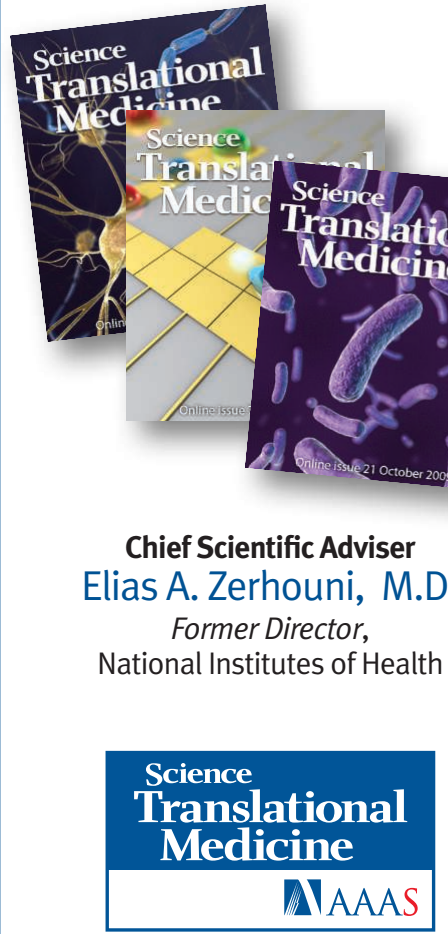

ScienceTranslationalMedicine.org 